\title{
SDF1 Regulates Leading Process Branching and Speed of Migrating Interneurons
}

\author{
Daniel E. Lysko, ${ }^{1}$ Mary Putt, ${ }^{2}$ and Jeffrey A. Golden ${ }^{1,3}$

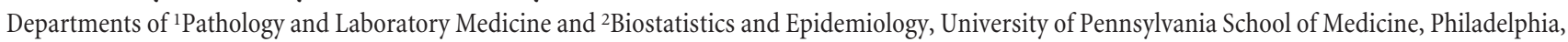 \\ Pennsylvania 19104, and 'Department of Pathology and Laboratory Medicine, Children's Hospital of Philadelphia, Philadelphia, Pennsylvania 19104
}

Cell migration is required for normal embryonic development, yet how cells navigate complex paths while integrating multiple guidance cues remains poorly understood. During brain development, interneurons migrate from the ventral ganglionic eminence to the cerebral cortex within several migratory streams. They must exit these streams to invade the cortical plate. While SDF1 (stromal cell-derived factor-1) signaling is necessary for normal interneuron stream migration, how they switch from tangential stream migration to invade the cortical plate is unknown. Here, we demonstrate that SDF1 signaling reduces interneuron branching frequency by reducing cAMP levels via a $G_{i}$ signaling pathway using an in vitro mouse explant system, resulting in the maintenance of stream migration. Blocking SDF1 signaling or increasing branching frequency results in stream exit and cortical plate invasion in mouse brain slices. These data support a novel model to understand how migrating interneurons switch from tangential migration to invade the cortical plate in which reducing SDF signaling increases leading process branching and slows the migration rate, permitting migrating interneurons to sense cortically directed guidance cues.

\section{Introduction}

Two primary pathways of migration exist for neurons destined for the cerebral cortex. Excitatory projection neurons migrate radially from the pallial ventricular zone (VZ) (Hatten, 2002; Kriegstein and Noctor, 2004). In contrast, interneurons (inhibitory neurons) have a much longer and more circuitous migration path, originating in the subpallial ganglionic eminence and migrating tangentially through the pallium, eventually turning to enter the cortical plate (Marín and Rubenstein, 2003). Interneurons predominately migrate within two defined streams: one in the marginal zone $(\mathrm{MZ})$ and the second a broader stream in the subventricular (SVZ) and intermediate (IZ) zones (McManus et al., 2004; Métin et al., 2006). Interneurons eventually turn out of these streams to enter the developing cortical plate, suggesting the presence of neocortical guidance factors that direct interneurons to their final position (supplemental Fig. S1, available at www.jneurosci.org as supplemental material) (López-Bendito et al., 2008).

Several secreted guidance factors have been identified for their roles in directing interneurons along their migratory path. For example, Sema3a repels migrating interneurons from the striatum (Tamamaki et al., 2003), while Nrg1-Ig attracts interneurons to migrate dorsally from the subpallium into the pallium (Flames

\footnotetext{
Received June 17, 2010; revised 0ct. 22, 2010; accepted Nov. 24, 2010.

This work was supported by National Institutes of Health Grants NS45034 and HD26979. We thank the members of the Golden laboratory for their helpful comments and support. We thank Dr. Jonathen Raper for the kind gifts of SDF1a and $\mathrm{G}_{\mathrm{i}}$ inhibitory expression vectors. We thank Gaia Colasante and Dr. Vania Broccoli for the kind gift of an SDF1a vector. We thank Dr. Ramon Pla, Juan Antonio Sanchez, and Dr. Oscar Marin for assistance with statistical analysis. We thank Drs. Jonathan Raper and Greg Bashaw for critically reviewing this manuscript.

Correspondence should be addressed to Jeffrey A. Golden, Children's Hospital of Philadelphia, 3615 Civic Center Boulevard., Abramson Research Center, Room 516G, Philadelphia, PA 19104. E-mail: goldenj@mail.med.upenn.edu. DOI:10.1523/JNEUROSCI.3118-10.2011

Copyright $\odot 2011$ the authors $\quad 0270-6474 / 11 / 311739-07 \$ 15.00 / 0$
}

et al., 2004). SDF1 (stromal cell-derived factor-1; also called CXCL12) was recently identified as a molecule necessary for normal stream migration (Stumm et al., 2003). SDF1 is a chemokine that induces chemotaxis in lymphocytes and is expressed in the cerebral cortex along the migration stream of interneurons, whereas the SDF1 receptor CXCR4 is expressed on migrating interneurons (Stumm and Hollt, 2007; Stumm et al., 2007; López-Bendito et al., 2008). In CXCR4-null mice stream migration is disrupted, causing interneurons to prematurely exit the migratory streams and invade the cortical plate, resulting in abnormal interneuron distribution (Li et al., 2008; López-Bendito et al., 2008). While these studies indicate that SDF1 signaling is necessary for normal interneuron distribution in the brain, it remains unknown how the loss of SDF1 signaling results in interneurons leaving their tangential trajectory and prematurely invading the cortical plate.

We and others have hypothesized that the leading process of migrating interneurons functions like an axon's growth cone to provide guidance during cell migration (Tessier-Lavigne and Goodman, 1996; Nasrallah et al., 2006; Martini et al., 2009; Gopal et al., 2010). Interneurons have a unique migratory behavior in which the nucleus moves in a saltatory manner behind a long single or branched projection: the leading process (Métin et al., 2006; Nasrallah et al., 2006). The leading process branches repeatedly during migration, possibly to sample the surrounding environment for guidance factors (supplemental Fig. S1D). Recent data demonstrating that interneurons preferentially branch toward attractants such as Nrg1 support this hypothesis (Martini et al., 2009). In this study, we test the hypothesis that SDF1 signaling influences interneuron branching to control interneuron stream migration and stream exit. We find that SDF1 suppresses branching, thus keeping migrating interneurons within their mi- 
gratory stream and moving rapidly. When SDF1 signaling is reduced, interneuron branching is stimulated, the rate of migration is slowed, and interneurons more frequently turn and exit their migratory streams.

\section{Materials and Methods}

Mouse strains. CD1 mice or CD1 mice crossed to Dlx5/6-Cre-IRES-eGFP mice (Stenman et al., 2003) to genetically label interneurons derived from the medial ganglionic eminence (MGE) with green fluorescent protein (GFP) were used for all experiments. All studies were approved by the Institutional Animal Care and Use Committee of the Children's Hospital of Philadelphia, Philadelphia, PA.

Slice and explant culture. Brains from embryonic day 14.5 (E14.5) mouse embryos were dissected in ice-cold HBSS (Sigma), embedded in $4 \%$ low melting point agarose (Lonza) in HBSS and sectioned coronally at $250 \mu \mathrm{m}$. Slices were placed on Millicell-CM culture inserts (Millipore), while $100 \mu \mathrm{m}$ square explants were cut out of the MGE of the slice and placed on plastic dishes or glass coverslips. The explants were covered with $50 \%$ Matrigel and 50\% collagen ( $2 \mathrm{mg} / \mathrm{ml}$; BD Biosciences) and placed at $37^{\circ} \mathrm{C}$ with $5 \% \mathrm{CO}_{2}$ for $20 \mathrm{~min}$ to gel. Tissue was incubated in DFS medium (F12:DMEM with $10 \% \mathrm{FBS}, 40 \mu \mathrm{M}$ L-glutamine, $47 \mathrm{~mm}$ glucose, and penicillin/streptomycin) for $1 \mathrm{~h}$, then switched to DM medium (DMEM with N2, 36 $\mathrm{mm}$ glucose, and penicillin/streptomycin). Slices were imaged after $1 \mathrm{~h}$ in DM medium, while explants were imaged after one day.

Microscopy. Speed and branching analyses were performed on time-lapse images acquired at $10 \times$ magnification and at $10 \mathrm{~min}$ intervals for $8-12 \mathrm{~h}$ on a Nikon TE300 microscope equipped with an Okolab environmental chamber at $37^{\circ} \mathrm{C}$ with $5 \% \mathrm{CO}_{2}$. Time-lapse imaging of GFP-labeled interneurons migrating in brain slices was acquired with $5 \mu \mathrm{m} Z$-stacks at $20 \times$ at $10 \mathrm{~min}$ intervals for $3.5 \mathrm{~h}$ on a Leica SP2 or Olympus FV10i confocal microscope. SDF1 bead-implanted slices were imaged on a Leica MZ16FA stereomicroscope.

Treatment protocols. Interneurons were exposed to SDF1a by coculturing COS7 cell clumps coexpressing SDF1a and DsRed 300 $\mu \mathrm{m}$ from MGE explants within Matrigel:collagen (BD Biosciences). COS7 cells were transiently transfected with FuGENE 6 (Roche) 2 d before pelleting and resuspension in an equal volume of Matrigel:collagen to form clumps. To inhibit $G_{i}$ signaling, slices were electroporated with a construct expressing interfering peptide IKNNLKDCGLF (cue BIOtech), followed by setting up explants as described above. Modulation of cAMP was achieved by treating tissue with $125 \mu \mathrm{M}$ forskolin (Sigma) or $790 \mu \mathrm{M}$ dibromo (db)-cAMP (Sigma) and imaged immediately. To block SDF1 signaling, slices were treated with the CXCR4 inhibitor AMD3100 (Sigma; $189.6 \mu \mathrm{M}$ ). To treat slices with BSA or recombinant SDF1 (PeproTech), agarose beads (Affi-Gel, Bio-Rad) were soaked
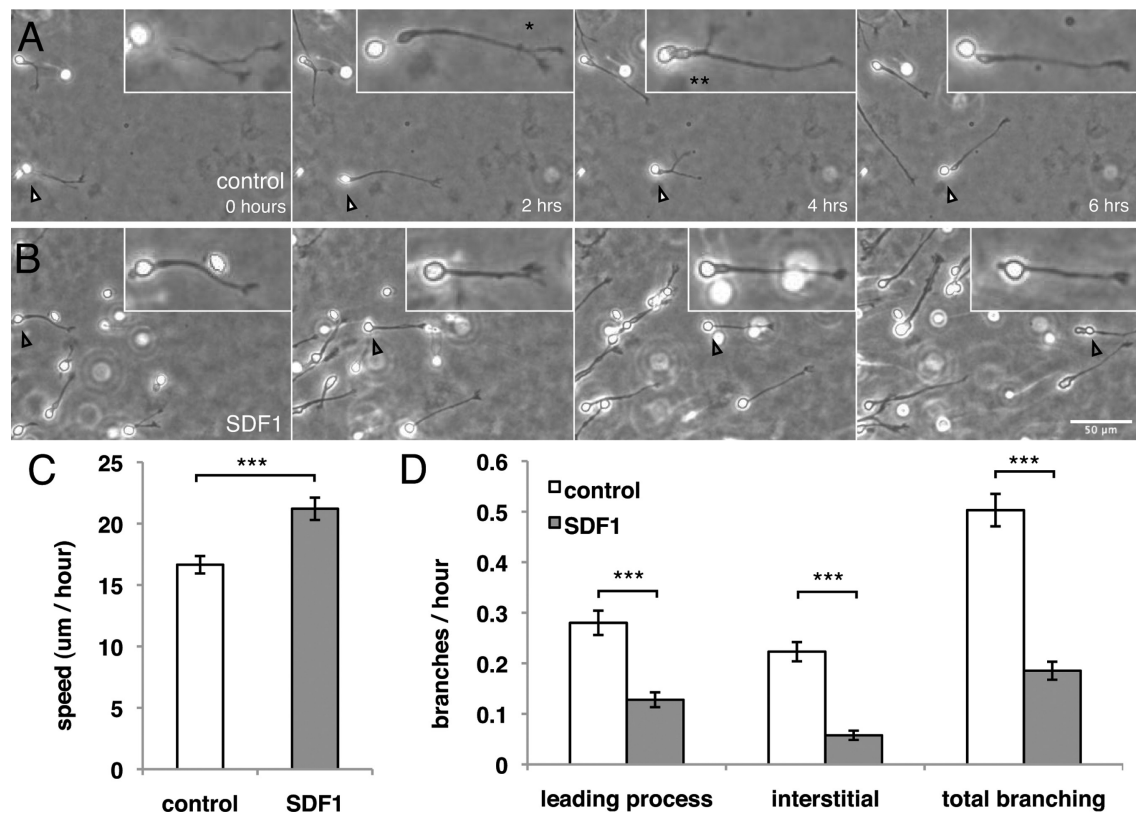

Figure 1. SDF1 reduces branching frequency. $\boldsymbol{A}$, Phase microscopy of migrating interneurons exhibiting leading process branching $\left({ }^{*}\right)$, interstitial branching $\left(^{* *}\right)$, and somal translocation (arrowheads) in control neurons. $\boldsymbol{B}$ (and supplemental Fig. 1, video 1), SDF1-treated interneurons show decreased branching frequency and faster somal translocation. C, D, Quantification of speed and branching of individual interneurons treated with SDF1 shows an increase in speed and a decrease in both leading process splitting and interstitial branching. Error bars are \pm SEM ( ${ }^{* *} p<0.001, t$ test); 45 cells for each condition; three independent experiments. Scale bar, $50 \mu \mathrm{m}$.
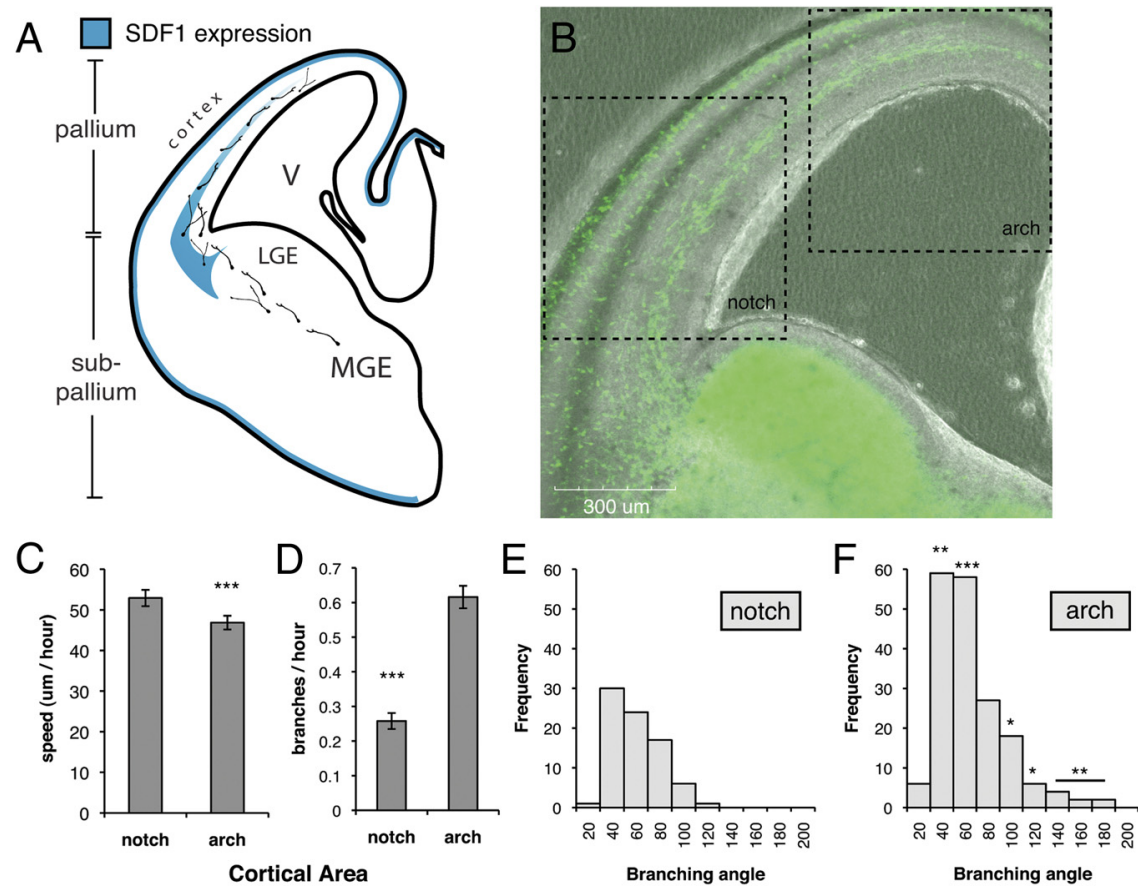

Figure 2. Interneurons in the SVZ/IZ migration stream decrease speed and increase branching as they migrate down the SDF1 expression gradient. $A$, SDF1 is expressed in two areas in the developing mouse brain: a narrow band near the surface of the brain and in a gradient within the cortex. These two areas are also the sites of interneuron stream migration seen in $\boldsymbol{B}$. LGE, Lateral ganglionic eminence; $V$, lateral ventricle. $\boldsymbol{B}$, Phase/confocal microscopy of GFP ${ }^{+}$interneurons migrating in a mouse brain slice, showing areas defined for analysis. $\boldsymbol{C}, \boldsymbol{D}$, Near the cortical notch, an area of high endogenous SDF1, interneurons migrate at $53 \pm$ $2 \mu \mathrm{m} / \mathrm{h}$ and branch infrequently $(0.26 \pm 0.02$ branches $/ \mathrm{h})$. Once in the arch area, an area of lower SDF1 expression, interneurons slow down ( $47 \pm 2 \mu \mathrm{m} / \mathrm{h}$ ) and branch more frequently ( $0.6 \pm 0.03$ branches $/ \mathrm{h}) . \boldsymbol{E}, \boldsymbol{F}$, Histograms of branch angle frequency show modest branch angles near the notch area, while in the arch area larger branch angles occur. Mean angles: $50 \pm 2.2$ (notch), $56 \pm$ 2.2 (arch). Error bars are \pm SEM $\left({ }^{* * *} p<0.001,{ }^{* *} p<0.01,{ }^{*} p<0.05, t\right.$ test or $\chi^{2}$ test in $\left.\boldsymbol{F}\right) ; 90$ cells for each condition; three independent experiments. Scale bar, $300 \mu \mathrm{m}$. 

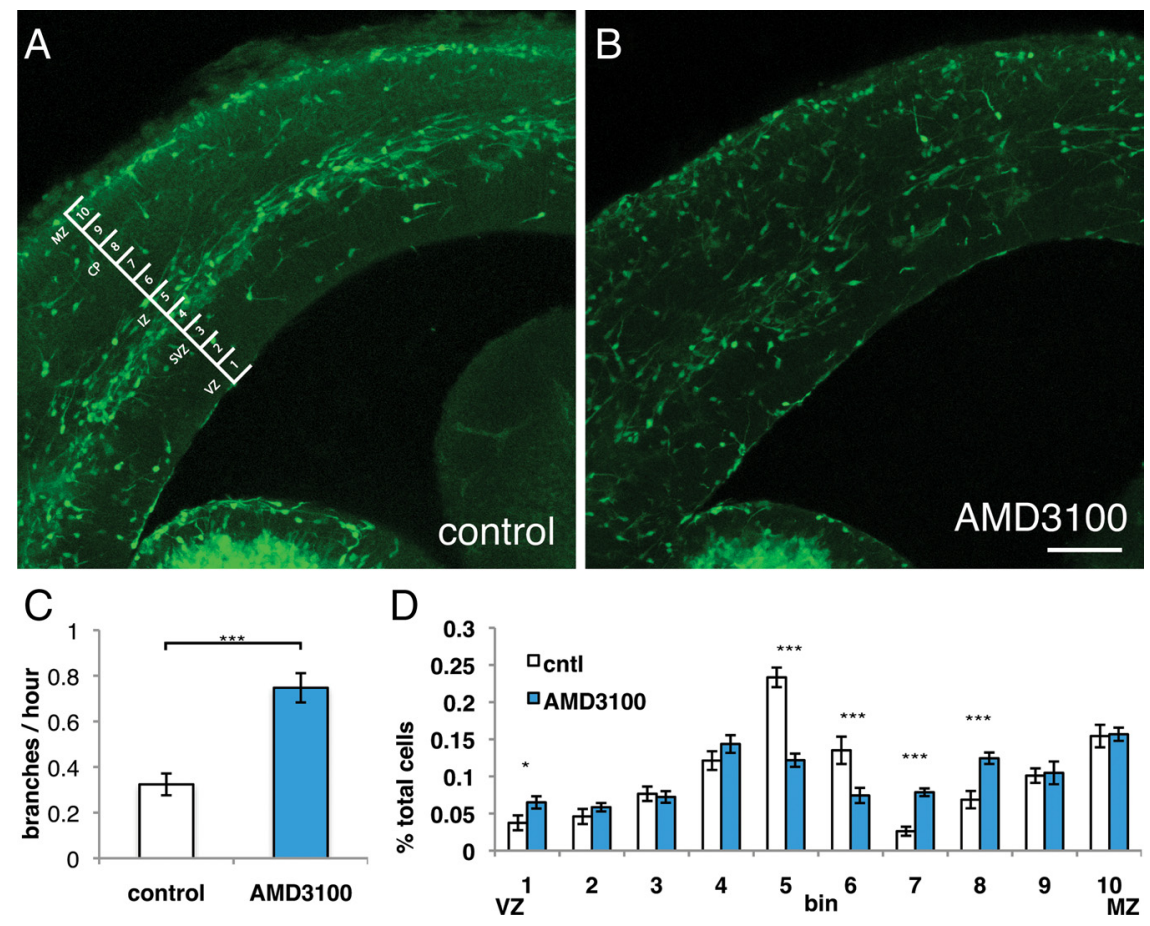

Figure 3. Blocking SDF1 signaling in brain slices increases branching and shifts interneuron distribution toward the cortical plate (CP). $\boldsymbol{A}$, Control-treated DIx5/6-Cre-IRES-GFP animals express GFP in interneurons born in the medial and lateral ganglionic eminences. The brain slice in $\boldsymbol{A}$ shows typical stream migration in the marginal zone, $\mathrm{MZ}$, and subventricular zone/intermediate zone, SVZ/IZ. B (and supplemental Figs. 3, 5, video 1), Blocking SDF signaling with AMD3100 results in a loss of distinct interneuron stream migration and an increase in interneurons in the cortical plate. C, Quantification of interneuron branching frequency shows a significant increase in branching in slices when SDF1 signaling is blocked. Error bars are \pm SEM $\left({ }^{* * *} p<0.001, t\right.$ test $)$; 18 cells for each condition; six independent experiments. $\boldsymbol{D}$, Interneurons normally contained in the SVZ/IZ migration stream (bins 5 and 6) are shifted outwards toward the cortical plate (bins 7 and 8 ) in slices with blocked SDF1 signaling. Several interneurons also migrated toward the VZ (bin 1) in AMD3100-treated slices. Error bars are $\pm \operatorname{SEM}\left({ }^{*} p<0.05,{ }^{* *} p<0.01,{ }^{* * *} p<0.001, \chi^{2}\right.$ test); 10 slices for each condition; three independent experiments; cntl, Control. Scale bar, $100 \mu \mathrm{m}$.

in $20 \mu \mathrm{g} / \mathrm{ml}$ protein solution $1-24 \mathrm{~h}$ at $4^{\circ} \mathrm{C}$ and imbedded into the slice at defined positions.

Quantification. Interneuron average speed was measured by recording cell soma position in each frame using the Manual Tracking plugin in ImageJ (National Institutes of Health; http://rsb.info.nih.gov/ij/) and averaging the speed from each frame in the interval of analysis. Interneuron branching was recorded manually. Interneurons with blocked $\mathrm{G}_{\mathrm{i}}$ signaling were identified by the fluorescence of cotransfected DsRed. Unfortunately, the fluorescence was not bright enough to allow reliable quantification of branching; speed was analyzed instead. Interneuron distribution across the neocortex was determined by manual counting using the Cell Counter plugin in ImageJ. Cells were counted in a defined 400 pixel-wide $(293 \mu \mathrm{m})$ area of analysis divided into 10 equal bins placed $300 \mu \mathrm{m}$ dorsal to the pallial/subpallial boundary.

Statistical analysis. Statistical analysis was performed using Microsoft Excel or R (www.r-project.org) with plugin R commander. If data appeared normally distributed, we asked whether there were differences between groups using multiway ANOVA and post hoc tests adjusted for multiple comparisons using a Bonferroni correction. For forskolin and $\mathrm{db}$-cAMP treatment in vitro the branching data appeared non-normally distributed so a similar approach was used, but in this case a generalized linear model with a Poisson distribution and a term to correct for overdispersion was used to fit the data, yielding an overall test of significance based on analysis of deviance. Interneuron distribution in AMD3100treated and forskolin-treated slices and branch angle distributions were analyzed using a $\chi^{2}$ test. If a difference was found between the populations, control and treated values for each bin were independently analyzed using $2 \times 2$ contingency tables.

\section{Results}

SDF1 reduces branching frequency in migrating interneurons

SDF1 signaling is necessary for normal interneuron stream migration, although the effect of SDF1 on interneuron migratory behavior is incompletely understood. In contrast, Nrg1 is known to alter interneuron branching to attract interneurons toward the pallium. We have hypothesized that SDF1 also influences branching behavior to facilitate interneuron migration within streams.

We examined the branching behavior of control-treated and SDF1-treated interneurons in vitro by time-lapse microscopy (explant culture). Interneurons treated with SDF1 migrate faster than controls, as reported previously (LópezBendito et al., 2008), but were also found to branch less frequently (Fig. 1). The SDF1-induced branching phenotype was found for both leading process splitting as well as interstitial branching (Fig. 1D). These data demonstrate that SDF1 signaling enhances the rate of migration while simultaneously affecting specific branching behavior: a reduction in branching frequency.

In vivo, stream migration occurs in areas of SDF1 expression (Stumm et al., 2007; López-Bendito et al., 2008); thus, we hypothesized that interneurons migrating within SDF1-rich streams into the pallium would exhibit reduced branching and fast migratory rates. As these neurons move from ventral to dorsal in the SVZ/IZ stream, they are exposed to progressively lower SDF1 levels (Fig. $2 A)$; thus, we predicted they would exhibit increased branching and slower migration. This would lead to increased exploration of their environment, increased directional changes, and ultimately accelerated cortical plate invasion.

To test this model in brain slices, we measured interneuron speed and branching frequency within the SVZ/IZ stream in two positions of differing SDF1 expression by time-lapse microscopy (Fig. 2B). The first position was just above the cortical notch where SDF1 is highly expressed (ventrolateral neocortex). Interneurons were found to branch at a modest rate $(0.24$ branches $/ \mathrm{h})$ and migrate quickly $(53 \mu \mathrm{m} / \mathrm{h}$ ) (Fig. $2 C, D)$. In contrast, farther up the cortical arch where SDF1 expression is lower and narrower (dorsomedial neocortex), interneurons branch $\sim 2.5$ times more frequently ( 0.6 branches/h) and slow down $(47 \mu \mathrm{m} / \mathrm{h})$. We also measured the angle between leading process branches, as differences in branch angle have been observed previously at different areas along the interneuron migration path (Martini et al., 2009). Interneurons migrating in the SVZ/IZ stream near the notch had an average branch angle of $50 \pm 2.2^{\circ}$, while interneurons migrating farther along in the arch had a slightly higher average branch angle of $56 \pm 2.2^{\circ}$. The distribution of branch angles in both areas appeared log normal, but only in the arch area did branch angles larger than $105^{\circ}$ occur, with significantly wider angles occurring throughout the distribution (Fig. 2E,F). 
Blocking SDF1 signaling increases interneuron branching in brain slices, driving interneurons from the SVZ/IZ migration stream to the cortical plate

To test the pathfinding aspect of our model, we acutely inhibited interneuron SDF1 signaling in brain slices. As expected and seen previously, inhibition of SDF1 signaling with the CXCR4 inhibitor AMD3100 disrupted migration stream organization similar to the phenotype of the CXCR4-null mouse (Fig. $3 A, B$ ) (LópezBendito et al., 2008). Importantly, inhibiting SDF1 signaling increased the branching frequency of interneuron leading processes in the SVZ/IZ migration stream (Fig. 3C) and resulted in a shift in interneuron distribution. Interneurons in control slices were most frequently present in two main migration streams (SVZ/IZ and MZ), whereas interneurons in AMD3100treated slices exited out of the SVZ/IZ migratory stream to find positions in the cortical plate and VZ (Fig. 3D). Thus, we find SDF1 signaling reduces branching frequency of migrating interneurons both in explant cultures and in brain slices. These data suggest that SDF1 induces a specific branching behavior in interneurons that is necessary for normal stream migration and interneuron position within the developing cortex.

\section{SDF1 uses a $\mathrm{G}_{\mathrm{i}}$-coupled signal transduction pathway to decrease interneuron branching}

The SDF1 signaling pathway governing this branching behavior has yet to be investigated in migrating interneurons, but SDF1 signaling is known to positively modulate leukocyte chemotaxis and primordial germ cell migration in zebrafish (Phillips and Ager, 2002; Knaut et al., 2003). The SDF1 receptor CXCR4 functions as a G-coupled protein receptor that can act through $\mathrm{G}_{\mathrm{i}}$ to inhibit adenylyl cyclase (AC), lowering cAMP levels and protein kinase A (PKA) activity. Given that other migrating cell types employ similar signaling mechanisms during migration, we predicted that the increased speed of migration and reduction in branching frequency seen in SDF1-treated interneurons in vitro would be the result of a similar signaling pathway.

To test this prediction, we electroporated interneurons in vitro with a cue BIOtech $G_{i}$ minigene vector expressing an interfering peptide known to specifically block $G_{i}$ signaling (explant culture). The $G_{i}$ minigene had little effect on migration in the absence of SDF1, but in the presence of SDF1 blocking $\mathrm{G}_{\mathrm{i}}$ signaling blocked the expected increase in migration rate (Fig. $4 \mathrm{~A}$ ).

Given that SDF1 signaling normally inhibits AC through $G_{i}$, we expected stimulation of AC to block the reduction in branching frequency seen in SDF1-treated interneurons. Stimulation of AC with forskolin increased branching frequency in controltreated neurons and blocked reduction of branching in SDF1treated interneurons (Fig. 4B). Similarly, stimulating protein kinase A with dibromo-cAMP also resulted in an increased branching frequency, but in the presence of SDF1 dibromocAMP blocks the expected reduction in branching (Fig. 4C). These data indicate that SDF1 inhibits branching of interneurons through this $\mathrm{G}_{\mathrm{i}}$-coupled receptor signal transduction pathway.

\section{Forskolin increases interneuron branching in brain slices, driving interneurons from the SVZ/IZ migration stream to the cortical plate}

To further test the sufficiency of increased branching to induce the same shift in interneuron distribution seen when inhibiting SDF1 signaling, we again used our slice culture system. We acutely increased the frequency of interneuron branching in brain slices by adding forskolin, as was done in explant culture. This resulted in a similar disruption of the migration streams as
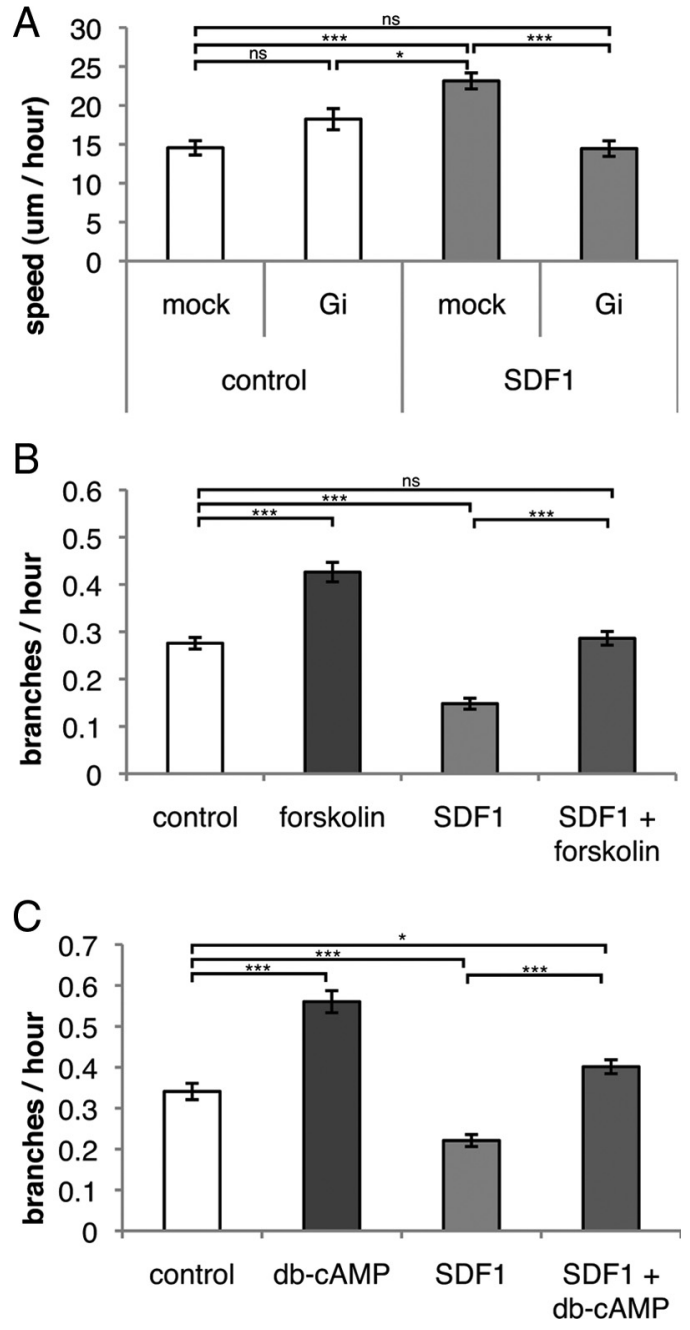

Figure 4. SDF1 affects branching frequency through a $G_{i}$-mediated mechanism. $A$ (and supplemental Fig. 4, video 1), Interneurons transfected with a $\mathrm{G}_{\mathrm{i}}$-specific interfering peptide migrate with similar speed as that of mock-treated cells in the absence of SDF1 (white bars, in vitro explant culture). SDF1 induces a faster migration speed in mock-treated cells, but this speed increase is blocked in cells with inhibited $\mathrm{G}_{\mathrm{i}}$ signaling (gray bars). Error bars are $\pm \mathrm{SEM}$ ( $p<$ 0.001 , multiway ANOVA, not significant (ns), $p>0.05,{ }^{*} p<0.05,{ }^{* * *} p<0.001, t$ tests with Bonferroni correction); $>32$ cells for each condition; three independent experiments. $\boldsymbol{B}$ (and supplemental Fig. 4, video 2), Stimulation of adenylyl cyclase with forskolin induces interneuron branching. SDF1 alone reduces branching, but this branching decrease is blocked when cells are also stimulated with forskolin. Error bars are \pm SEM ( $p<0.001$, analysis of deviance, ns, $p>0.05,{ }^{* * *} p<0.001$, post hoc tests with Bonferroni correction); $>70$ cells for each condition; three independent experiments. ( (and supplemental Fig. 4, video 2), Stimulation of protein kinase $A$ with db-CAMP induces interneuron branching. SDF1 alone reduces branching, but this branching decrease is blocked when cells are also stimulated with db-CAMP. Error bars are \pm SEM $\left(p<0.001\right.$, analysis of deviance, ${ }^{*} p<0.05,{ }^{* * *} p<0.001$, post hoc tests with Bonferroni correction); $>58$ cells for each condition; three independent experiments.

seen in brain slices with inhibited SDF1 signaling (compare Figs. $5 A, B, 3 A, B)$. As expected, this treatment increased the branching frequency of interneurons and resulted in a shift in interneuron distribution (Fig. 5C,D). Interneurons exited from the SVZ/IZ migration stream to positions in the cortical plate. Importantly, treatment with forskolin did not effect interneurons' capacity to respond to SDF1 in brain slices in a bead attraction assay (supplemental Fig S2, available at www.jneurosci.org as supplemental material), supporting our model that either inhibiting SDF1 signaling or increasing branching frequency is sufficient to allow interneurons to exit at least one of their migration streams and invade the cortical plate. 

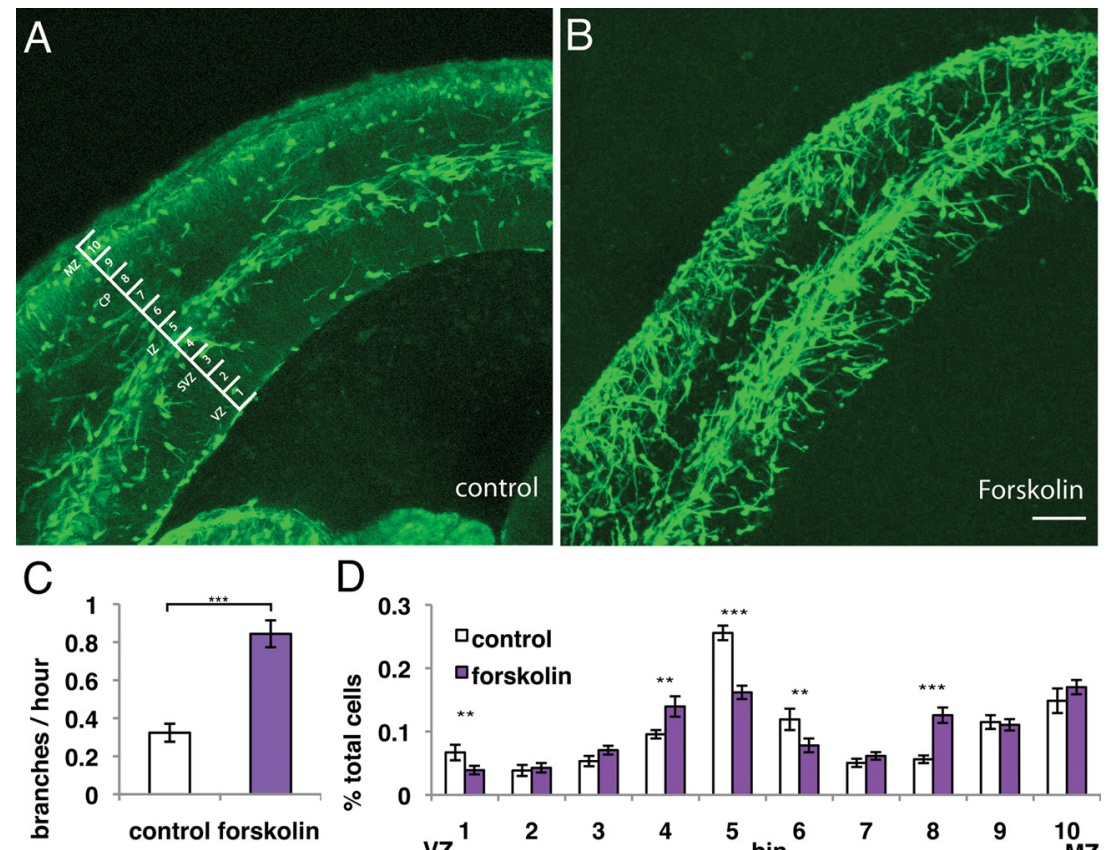

D

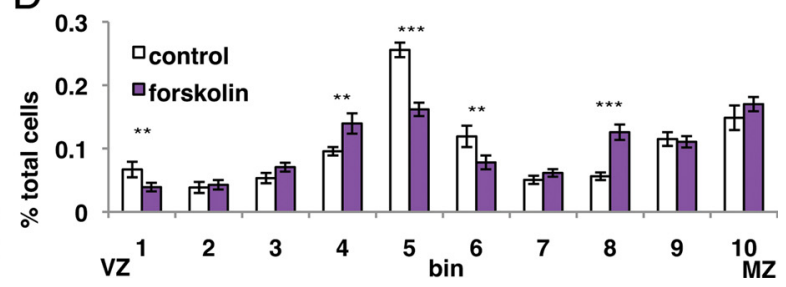

Figure 5. Increasing branching frequency in brain slices shifts interneuron distribution toward the cortical plate (CP). $A, B$ (and supplemental Figs. 3, 5, video 1), Normal stream migration is disrupted by increasing branching frequency with forskolin treatment similar to blocking SDF1 signaling (compare with Fig. 3). C, Quantification of branching frequency of interneurons in slices treated with forskolin shows a significant increase in branching frequency. Error bars are $\pm S E M\left({ }^{* * *} p<0.001, t\right.$ test); 18 cells for each condition; six independent experiments. $\boldsymbol{D}$, Interneurons normally contained in migration streams in bins 5 and 6 are shifted outwards toward the cortical plate (bins 7 and 8 ) in slices treated with forskolin. Error bars are $\pm \operatorname{SEM}\left({ }^{*} p<0.05,{ }^{* *} p<0.01\right.$, ${ }^{* * *} p<0.001, \chi^{2}$ test); $>9$ slices for each condition; seven independent experiments. Interneuron migration toward the VZ (bin 1) was not seen in forskolin-treated slices; some neurons may migrate away from the VZ in treated slices. Scale bar, $100 \mu \mathrm{m}$.

Interneuron leading process branching and migration rates are inversely regulated by SDF1 signaling

Interneurons in CXCR4-null mice prematurely exit their migration streams and migrate to the cortical plate, eventually exhibiting an abnormal distribution (Li et al., 2008; López-Bendito et al., 2008). Our data indicate that SDF1 increases the speed of migration while decreasing the frequency of branching. Based on these findings, we predicted that branching and speed would be coregulated by CAMP. To test this hypothesis, we treated interneurons with different concentrations of forskolin to induce branching and measured interneuron migration speed. We found that as branching frequency increases, migration speed decreases (Fig. 6). At the highest concentrations of forskolin we observed cells branching most frequently, while some cells also frequently change the polarity of their leading process and migrate quickly back and forth, resulting in a higher average speed. Cells treated with lower doses of forskolin branch more frequently than control cells while maintaining the polarity of their leading process, resulting in the lowest migration speed. These data support our model that intracellular signaling pathways that affect branching frequency through cAMP levels or PKA activity inversely affect speed.

\section{Discussion}

Understanding how migrating neurons navigate the complex environment of the embryonic brain is a fundamental question in CNS development. Although progress has been made in understanding how migrating cells move and change their direction in vitro, the mechanisms migrating cells use as they are directed through tissue by multiple guidance factors are largely unknown.

We have examined interneuron stream migration in the cortex and tested the role of SDF1 in stream maintenance and inter- neuron stream exit. Although SDF1 had previously been identified as a modulator of migration rate in vitro, data we were able to confirm, we also determined that SDF1 signaling reduces interneuron branching frequency both in vitro and in brain slices. In this context, inhibiting SDF1 signaling results in interneurons prematurely exiting from migratory streams and shifting the interneuron distribution from the SVZ/IZ to the cortical plate. We found that inducing branching in migrating interneurons is sufficient to cause cells to exit their migration stream and invade the cortical plate in brain slices. While treatment with AMD3100 will act autonomously on interneurons, we have not yet excluded the possibility that treatment with forskolin could act nonautonomously to cause cortical plate invasion. Together, our data suggest that SDF1 signaling suppresses branching frequency to allow cells to maintain a simpler morphology better suited to rapid migration within a defined stream. Interestingly, these treatments did not cause a measurable amount of interneurons to exit the MZ stream, where SDF1 is also highly but more uniformly expressed (Figs. 3, 5, bin 10). This could suggest intrinsic differences between the SDF1 response of interneurons occupying the two different migration streams, external substrate differences, or a more complex guidance switch with additional signaling factors to induce interneurons to exit the MZ to invade the cortical plate.

To characterize the intracellular signaling pathway used by SDF1 in interneurons, we interrogated known components of the pathway. We expected CXCR4 signaling to occur through $G_{i}$ as in other cell types. We found that $G_{i}$ regulation of cAMP concentration is important in defining interneuron speed and branching frequency. We also found that protein kinase A may transduce this second messenger signal to further signaling pathways to create the complex migration behavior of interneurons.

Our data imply an inverse relationship between branching and the rate of migration. Treating interneurons with SDF1 resulted in both an increase in speed and a decrease in leading process branching. We next asked whether regulation of these two aspects of cell behavior diverge farther down the SDF1 signaling pathway. Treatment over a range of forskolin concentrations, which increases cAMP through adenylyl cyclase sensitization, determined that increasing cAMP concentration increases branching while reducing speed. Together with our SDF1 treatment results, cAMP concentration is a central mediator of both speed and branching frequency. Whether cAMP affects speed and branching through separate downstream signaling pathways or through shared cytoskeletal regulation remains to be determined.

The inverse relationship between branching and speed has led us to propose a new model to describe interneuron stream migration (Fig. 6B). In this model, interneurons migrating in streams under the influence of SDF1 migrate faster as a result of a reduced branching frequency. This allows interneurons to spread 
from their ganglionic eminence origins through the cortex and achieve a proper interneuron distribution. As individual interneurons encounter lower SDF1 levels and consequently reduce SDF1 signaling, they increase their branching and reduce their speed. This permits them to sample as yet unknown cues, change direction, and migrate into the cortical plate to achieve their final position.

One possible cortical plate invasion cue could be a time-dependent reduction in SDF1 signaling levels. It is likely that SDF1 signaling within individual interneurons is reduced as they exit migration streams (Liapi et al., 2008; López-Bendito et al., 2008). Consistent with our observation of increased branching and stream exit when treating brain slices with SDF1 signaling inhibitor, migratory interneurons in the CXCR4 knock-out mouse exit their migration streams prematurely, demonstrating that a reduction in SDF1 signaling allows cells to exit migration streams, but also that SDF1 alone cannot define and maintain stream migration ( $\mathrm{Li}$ et al., 2008). Whether this reduced SDF1 signaling is the result of downregulation of CXCR4 or internally initiated interference with the SDF1 signaling pathway, perhaps at the cAMP level, remains to be elucidated. One external cue for interneuron stream exit is likely decreasing SDF1 concentration as interneurons migrate down the gradient of SDF1 expression (Stumm et al., 2007). Consistent with this hypothesis, our measurement of interneuron branching in brain slices shows that interneuron branching is lower near the cortical notch, an area of high SDF expression; but farther up the cortical arch where SDF expression is reduced, interneuron branching increases (Fig. 2).

According to our data and model, as SDF1 signaling is reduced, the branching frequency of interneurons increases (Fig. $6 B)$. We speculate that this increase in branching allows interneurons to probe greater space and come into contact with additional guidance factors. A cortical plate attractant has been biologically demonstrated, but is yet to be identified (LópezBendito et al., 2008). In addition to the cortical plate attractant, it is possible that repellents could direct interneuron migration in the cortex. There is some evidence that semaphorins may be expressed in the dorsal VZ/SVZ (Tamamaki et al., 2003), and SDF1 has been shown to reduce the response of pathfinding axons to repellents in the Slit and Semaphorin families (Chalasani et al., 2003). In the cortex, interneurons migrating in SDF1-rich streams would be insensitive to any repellents. Upon reduction in SDF1 signaling, interneurons could then respond to the repellents and exit migration streams toward the cortical plate. Interestingly, we provide some evidence that reduction of SDF1 signaling with AMD3100 can slightly increase interneuron localization in the VZ (Fig. 3, bin 1), suggesting that blocking SDF1 signaling may leave interneurons free to explore the VZ. In contrast, if SDF1 signaling is intact but interneuron branching is increased by forskolin treatment, fewer cells localize to the VZ even though cells exit the SVZ/IZ stream on both sides, possibly displaying a balance between SDF1's attractive ability and interneuron branching causing more exploratory migration (Fig. 5, bins 1 and 4-6). Finally, in our model increased branching would shift the balance of stimulation from SDF1 to the cortical plate attractant or a VZ-localized repellent, resulting in slower migration and stream exit. The role of SDF1 in this model is to affect pathfinding by modulating cell morphology, not necessarily for SDF1 to function as an attractant or a motogen, both additional possible functions (Li et al., 2008; López-Bendito et al., 2008).

Our data provide a new model as to how SDF1 controls interneuron stream migration through signal transduction and modification of cell migratory morphology and behavior. Because we have shown that changes in branching frequency are relevant in a tissue context, we expect future studies to further define the role of migrating interneuron branching in brain development.

\section{References}

Chalasani SH, Sabelko KA, Sunshine MJ, Littman DR, Raper JA (2003) A chemokine, SDF-1, reduces the effectiveness of multiple axonal repellents and is required for normal axon pathfinding. J Neurosci 23:1360-1371.

Flames N, Long JE, Garratt AN, Fischer TM, Gassmann M, Birchmeier C, Lai C, Rubenstein JL, Marín O (2004) Short- and long-range attraction of cortical GABAergic interneurons by neuregulin-1. Neuron 44:251-261.

Gopal PP, Simonet JC, Shapiro W, Golden JA (2010) Leading process 
branch instability in Lis $1^{+/-}$nonradially migrating interneurons. Cereb Cortex 20:1497-1505.

Hatten ME (2002) New directions in neuronal migration. Science 297: $1660-1663$.

Knaut H, Werz C, Geisler R, Nüsslein-Volhard C, Tübingen 2000 Screen Consortium (2003) A zebrafish homologue of the chemokine receptor Cxcr4 is a germ-cell guidance receptor. Nature 421:279-282.

Kriegstein AR, Noctor SC (2004) Patterns of neuronal migration in the embryonic cortex. Trends Neurosci 27:392-399.

Li G, Adesnik H, Li J, Long J, Nicoll RA, Rubenstein JL, Pleasure SJ (2008) Regional distribution of cortical interneurons and development of inhibitory tone are regulated by Cxcl12/Cxcr4 signaling. J Neurosci 28:1085-1098.

Liapi A, Pritchett J, Jones O, Fujii N, Parnavelas JG, Nadarajah B (2008) Stromal-derived factor 1 signalling regulates radial and tangential migration in the developing cerebral cortex. Dev Neurosci 30:117-131.

López-Bendito G, Sánchez-Alcañiz JA, Pla R, Borrell V, Picó E, Valdeolmillos M, Marín O (2008) Chemokine signaling controls intracortical migration and final distribution of GABAergic interneurons. J Neurosci 28:1613-1624.

Marín O, Rubenstein JL (2003) Cell migration in the forebrain. Annu Rev Neurosci 26:441-483.

Martini FJ, Valiente M, López Bendito G, Szabó G, Moya F, Valdeolmillos M, Marín O (2009) Biased selection of leading process branches mediates chemotaxis during tangential neuronal migration. Development 136:41-50.

McManus MF, Nasrallah IM, Gopal PP, Baek WS, Golden JA (2004) Axon mediated interneuron migration. J Neuropathol Exp Neurol 63:932-941.

Métin C, Baudoin JP, Rakić S, Parnavelas JG (2006) Cell and molecular mechanisms involved in the migration of cortical interneurons. Eur J Neurosci 23:894-900.

Nasrallah IM, McManus MF, Pancoast MM, Wynshaw-Boris A, Golden JA (2006) Analysis of non-radial interneuron migration dynamics and its disruption in Lis1 ${ }^{+/-}$mice. J Comp Neurol 496:847-858.

Phillips R, Ager A (2002) Activation of pertussis toxin-sensitive CXCL12 (SDF-1) receptors mediates transendothelial migration of T lymphocytes across lymph node high endothelial cells. Eur J Immunol 32:837-847.

Stenman J, Toresson H, Campbell K (2003) Identification of two distinct progenitor populations in the lateral ganglionic eminence: implications for striatal and olfactory bulb neurogenesis. J Neurosci 23:167-174.

Stumm R, Höllt V (2007) CXC chemokine receptor 4 regulates neuronal migration and axonal pathfinding in the developing nervous system: implications for neuronal regeneration in the adult brain. J Mol Endocrinol 38:377-382.

Stumm RK, Zhou C, Ara T, Lazarini F, Dubois-Dalcq M, Nagasawa T, Höllt V, Schulz S (2003) CXCR4 regulates interneuron migration in the developing neocortex. J Neurosci 23:5123-5130.

Stumm R, Kolodziej A, Schulz S, Kohtz JD, Höllt V (2007) Patterns of SDFlalpha and SDF-1gamma mRNAs, migration pathways, and phenotypes of CXCR4-expressing neurons in the developing rat telencephalon. J Comp Neurol 502:382-399.

Tamamaki N, Fujimori K, Nojyo Y, Kaneko T, Takauji R (2003) Evidence that Sema3A and Sema3F regulate the migration of GABAergic neurons in the developing neocortex. J Comp Neurol 455:238-248.

Tessier-Lavigne M, Goodman CS (1996) The molecular biology of axon guidance. Science 274:1123-1133. 